\title{
Research Progress on Tissue Engineering of Main Tissues and Organs of Human Body
}

\author{
Zhirui Jin $^{1}$ \\ ${ }^{1}$ College of Veterinary Medicine, Southwest University, Chongqing, 400715, China
}

\begin{abstract}
The injury and failure diseases of human tissues and organs, such as heart failure and chronic kidney disease, seriously threaten human health and life safety. At present, however, organ transplantation has obvious limitations, and tissue engineering is considered as a potential alternative therapy. Tissue engineering uses the construction of cells, biomaterials and bioreactors to develop three-dimensional artificial tissues and organs for the enhancement, repair and replacement of damaged or diseased tissues and organs, which contributes to the fundamental solutions of diseases of tissues and organs as well as to the improvement of human health. This paper introduces the research progress of tissue engineering technology in the field of living organs from three aspects: seed cells, application of growth factors and biomimetic preparation of functionalized scaffold materials, hoping to provide help and ideas for the research and industrial development of the repair and reconstruction of human organs.
\end{abstract}

\section{Background}

The damage and dysfunction of organ tissue caused by various causes are important factors affecting human health and life safety. Heart disease is one of the most important diseases threatening human health. Every year, a large number of people die from heart disease. According to statistics, about 22 million people worldwide are suffering from heart failure.[1] Chronic kidney disease, as a global public health problem, affects $8-16 \%$ of the global adult population. Current methods for the treatment of end-stage organ failure include autologous or allograft transplantation, xenotransplantation, and artificial substitutes. However, due to the rapid increase in demand for organ transplantation and the inadequate supply of organ donors, as well as the high cost of treatment, secondary trauma and immune rejection, there is still an urgent need for a new treatment that can make up for the deficiency of organ transplantation and effectively treat organ failure. In this severe situation, tissue engineering has come into being. Tissue engineering is a multidisciplinary field, involving stem cell technology, biotechnology, chemical materials and other fields, through the application of cell, biomaterial and bioreactor construction to develop three-dimensional artificial tissues and organs. This technique can be used not only to strengthen, repair and replace damaged or diseased tissues, but also to provide treatment for a number of persistent diseases, such as malignant tumors, diabetes, heart disease, Alzheimer's disease, Parkinson's disease, stroke and other diseases, with broad application prospects.

With the development of cell-directed differentiation, $3 \mathrm{D}$ bio-printing, biomaterials and tissue engineering reactors and other researches, many advances have been made in the regeneration of human tissues and organs, including bone[2], nerve[3], skin[4] and organs of heart, lung, liver, kidney, etc. Recently, the researchers reprogrammed the autologous cells to become them pluripotent stem cells, differentiated them into cardiomyocytes and endothelial cells, processed the extracellular matrix into hydrogels, and then combined the two cell types with hydrogels to form biological ink for parenchymal heart tissue and blood vessels. Then a functional myocardial patch with a personalized vascular pedicle was printed by 3D printing.[5] The researchers also designed an inverted colloidal crystal polymeric scaffold that customizes the matrix needed for different stages of cell development, thus better simulating the extracellular niche for the growth of liver buds, and thus used for the culture of liver buds, which is a good model for the study of liver disease.[6] In addition, the researchers successfully transformed mouse and human fibroblasts into induced renal tubular epithelial cells, which have epithelial characteristics and sensitivity to nephrotoxic substances, which can be used in disease modeling, drug and renal toxicity tests to promote organ regeneration.[7] These results show broad application prospects of tissue engineering and lay a foundation for the construction and transplantation of engineered organs in the future.

As a new potential alternative therapy, tissue engineering has attracted more and more researchers' attention, and people's in-depth research on tissue engineering of vital organs has also made a series of progress. This paper will introduce the frontier research and development of tissue engineering of vital organs from three aspects: seed cells, application of growth

e-mail: 1259170932@qq.com 
factors and biomimetic preparation of functionalized scaffold materials.

\section{Seed Cells}

Seed cells are the core elements of tissue engineering. Obtaining sufficient number of seed cells with regenerative vitality without immune rejection and making them expand and mature into functional cells in a targeted way is the cellular basis for repairing damaged organs and in vitro organ construction. At present, totipotent stem cells, pluripotent stem cells and directional stem cells are the most important seed cells in tissue engineering research. In addition to stem cells, transdifferentiation or lineage reprogramming can also transform endothelial cells[8], epithelial cells[9], fibroblast[10-13], muscle cells[14], tendon cells[15], hepatocyte[16] and other differentiated cells with limited ability to divide into other types of functional cells or progenitor cells, and use them for tissue engineering research. Duncan et al.[17] differentiated induced pluripotent stem cells into hepatocytes by adding five growth factors. This study provides possibility for efficient production of patient-specific hepatocytes, and can also be used for the study of hepatocyte disease and basic developmental mechanisms as well as drug screening. By co-expressing four transcription factors, Emx2, Hnf1b, Hnf4a and Pax8, Kaminski et al.[7] transformed mouse and human fibroblasts into induced tubular epithelial cells. This study is helpful in facilitating disease modeling, drug and renal toxicity testing, and lays the foundation for organ regeneration.

In order to avoid immune rejection and other problems, autologous cells are often used as seed cells in tissue engineering research to repair tissue damage. However, it is difficult for autologous tissue cells to reach the number and concentration required for cell culture, and the problem of cell function aging after long-term transmission also limits the application of autologous cells. In addition, the autologous cells of patients with congenital diseases have immunodeficiency or genetic defect, so it is necessary to select the seed cells from allogeneic sources for effective treatment. With the development of research on seed cells, the researchers have found that human amniotic mesenchymal stem cells, placental mesenchymal stem cells, bone marrow mesenchymal stem cells and adipose mesenchymal stem cells have the characteristics of low immunogenicity, which can inhibit the proliferation and function of major immune cell groups, including $\mathrm{T}$ cells, and they are also easy to isolate and culture, and have pluripotent differentiation.[18] Therefore, they are not easily recognized by the host immune system and can be applied to allograft or xenograft. Thus, they are potential and valuable seed cells. By studying several kinds of mesenchymal cells, the researchers found that bone marrow mesenchymal stem cells and adipose mesenchymal stem cells have the ability of long-term culture in vitro and the differentiation potential of trisomy (osteogenesis, adipogenesis and chondrogenesis), and can significantly inhibit the proliferation of allogeneic T cells, therefore they are excellent sources of seed cells for tissue engineering and regenerative medicine.[19] The researchers used nicotinamide, 1-taurine and sodium butyrate in high glucose medium to differentiate bone marrow mesenchymal stem cells into pancreatic progenitor cells, and then combined nicotinamide with exendin-4 or GLP-1 to eventually differentiate it into beta cells. Compared with pluripotent stem cells, mesenchymal stem cells have less toxic or residual effects on tumorigenicity and small molecules when treated as seed cells and can be used in the treatment of diabetes.[20] Human amniotic mesenchymal stem cells can reduce immune responses in allograft models in vitro, indicating the potential value of such cells in the treatment of premature newborns and post-transplant treatment.[21] In addition, mesenchymal stem cells can be used to treat multiple sclerosis[22], Duchenne muscular dystrophy[23], tumor disease[24], and acute pancreatitis[25].

Although there are still some problems in seed cells, such as limited source, low amplification and differentiation efficiency, these problems may be well solved in the future with the extensive research and indepth exploration of seed cells.

\section{Growth Factors}

Growth factor (GF) is small molecular substance with biological effects such as inducing and stimulating cell proliferation and differentiation, maintaining cell survival, and plays an important role in cell proliferation, repair and regeneration of tissues or organs. It is also able to improve cell reprogramming efficiency[26] and is a key component of tissue engineering and regenerative medicine. In the study of tissue engineering, various growth factors are often loaded on matrix materials and released to seed cells quantitatively and continuously, which can not only promote reprogramming of transcription factors by modulating signal pathways[27-29], regulating epigenetic barriers [30, 31] and regulating metabolism[32, 33], so as to induce the desired cell types in vitro, but also be used as drugs to promote the regeneration of endogenous cells in patients. The small molecule growth factors have the advantages of low cost, easy for synthesis, preservation and curing, which can quickly produce reversible biological effects, and at the same time, make up for the insecurity and low induction efficiency of exogenous genes[34]. With the development of the research on growth factors, more and more researches have been successfully carried out to obtain other types of functional cells based on transcriptional factor or small molecule compound-mediated method. Through CHIR99021, A8301, BIX01294, AS8351, SC1, Y27632, OAC2, SU16F and JNJ10198409, Nan Cao et al.[11] realized the reprogramming of human fibroblasts into cardiomyocytes, which provided basis for in situ cardiac repair by targeting endogenous cardiac fibroblasts with small molecules. Tang et al. [35] transformed mouse fibroblasts directly into neural stem cells without hypoxia through valproic acid, CHIR99021 and Repsox in the absence of hypoxia. Jin et al.[36] used Forskolin, DAPT, morphine and a transcription factor SF1 to transform human fibroblasts 
into functional Leydig-like cells, laying the foundation for the treatment of male primary gonadal dysfunction through Leydig cell transplantation. Wang et al. [9] successfully transformed human gastric epithelial cells into pluripotent endoderm progenitor cells through signal pathway inhibitors SB431542, Ca 2+ channel activator Bay K8644, epigenetic regulators Bix01294 and RG108. Liu et al.[37] used CHIR99021, LDN193189, A83-01, Hh-Ag1.5, retinoic acid (RA), SMER28, RG108, Parnate and basic fibroblast growth factor (bFGF) to transform mouse fibroblasts into oligodendrocytes, providing potential therapeutic strategies for the treatment of demyelinating diseases. Kim et al.[38] successfully reprogrammed human liver cells into dual-energy progenitor cells through A83-01 and CHIR99021. This method can generate a large number of patient-specific liver progenitor cells, opening up a new way for the development of cell-based patient-specific regenerative medicine treatment strategies. Ma et al.[39] effectively reprogrammed cultured human fetal astrocytes into functional neurons using CHIR99021, D-APT, LDN193189 and SB431542, which is helpful to further improve the potential chemotherapy for nerve regeneration and repair. By exogenous adenosine induction, Kang et al.[40] cultured human pluripotent stem cells in osteogenic induction medium containing glycerophosphate, ascorbic acid 2-phosphate and dexamethasone to convert them directly into functional osteoblasts. This study is the first to realize the direct transformation of human pluripotent stem cells into osteoblasts by a single small molecule, which will help to obtain a large number of regenerative cells to treat severe bone defects.

At present, the application of growth factor still has some problems, such as low efficiency, drug toxicity, and some side effects, such as when the growth factor is injected into the injured part of the brain, it may induce changes in normal neurons. However, with the development of research, the mechanism of growth factors will be further elucidated, which will contribute to ensure the safety of its clinical application and optimize the efficient induction of functional cells without exogenous gene integration.

\section{Scaffold Material}

Scaffold refers to the three-dimensional structure that seed cells rely on for survival and attachment before they regenerate tissues during tissue and organ reconstruction. It is mainly made of biodegradable and absorbable biological materials and is a biomimetic extracellular matrix. Bionic scaffold materials can not only provide temporary mechanical support for cell growth and necessary growth micro-environment, but also provide a good inducement for cell specific differentiation and a place for seed cells to adhere, grow and differentiate.

According to different sources, scaffold materials can be divided into three types: natural extracellular matrix materials, synthetic materials and composite materials. Natural extracellular matrix materials are polymers or inorganic materials derived from organisms and their modified materials, such as collagen, gelatin, fibrin, alginate, dextran, glycosaminoglycan, etc. This material has good histocompatibility, no obvious toxicity, can be eventually degraded into polysaccharides or amino acids and absorbed by the body, and it is not easy to cause inflammatory reaction and immune reaction, but it has some disadvantages such as poor mechanical strength and difficult to control the degradation time[41]. As a result, more and more researchers have focused on synthetic materials and composites. The synthetic materials, including polylactide (PLA), polyethyl ester (PGA), polyethyl lactone (PCL), etc., have better mechanical properties and controllability to degradation rate. However, these materials have low bioactivity and lack of signal transduction proteins and their receptors or ligands that interact with seed cells, resulting in weak cell affinity and poor cell adhesion[42]. Composite material is a new material which combines 2 or more different kinds of materials in a certain proportion and method, which can overcome the deficiency of using a single material, such as hydroxyapatite-collagen composite material[43, 44] and collagen-PLCL composite scaffold[45, 46].

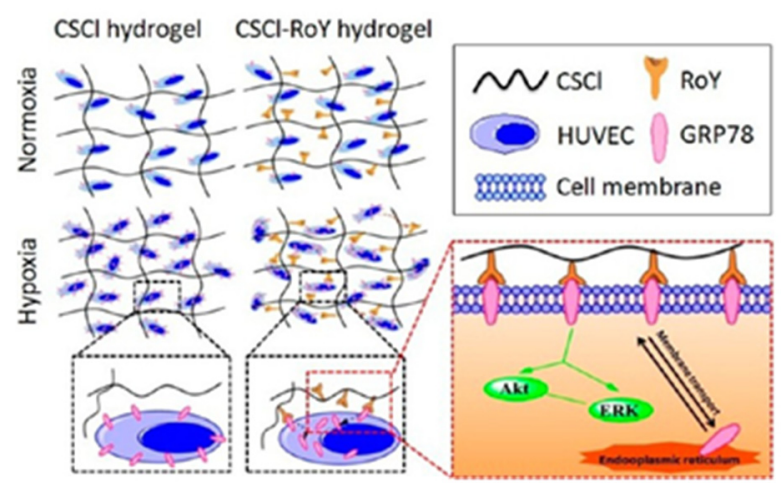

Fig. 1. CSCl-RoY as a scaffold material for cardiac tissue engineering[47]

With the development of research, researchers have made a lot of progress in functionalized biomimetic scaffold materials. Wu et al.[48] successfully constructed the endothelial myocardium by simulating the structure of natural heart tissue with a three-dimensional hybrid scaffold composed of polycaprolactone, silk fibroin and carbon nanotubes. The results showed that these scaffolds could promote the orientation and elongation of cytoplasm in each layer and control the orientation of cells in different layers, providing a method for guiding the growth and remodeling of cell orientation. Shu[47] et al. found that THE SCL-Roy hydrogel can promote survival, proliferation, migration and tubular formation of human umbilical vein endothelial cells under hypoxia conditions, improve angiogenesis in myocardial infarction model and improve myocardial function, and is an ideal scaffold for heart tissue engineering (Fig. 1). Lih et al.[49] successfully repaired and reconstructed glomeruli and blood vessels using renal extracellular matrix containing polylactic acid-glycolic acid (PLGA) scaffold. This method can not only be used as an effective alternative for the treatment of kidney diseases, but also shows the potential application of PLGA scaffold in biomedical 
fields such as tissue engineering biomimetic scaffolds and biodegradable implants. Shilpa et al. [50], through the proliferation and osteogenesis of dental pulp stem cells on hydroxyapatite-collagen ( $\mathrm{HA}-\mathrm{Col})$ scaffold, proved that the scaffold has no cytotoxicity, and has good biocompatibility, easy adhesion and proliferation of mesenchymal stem cells in vitro, and can enhance the proliferation and osteogenesis of DPSC, is an ideal material for maxillofacial and alveolar bone regeneration. $\mathrm{Wu}$ et al.[51] made heparinized acellular porcine liver scaffold by terminal attachment technology, which can bind and slowly release heparin binding growth factors. Compared with natural scaffolds, this scaffold can significantly and rapidly promote angiogenesis. Zhou et al.[52] implanted PCL scaffold containing active Schwann cells and neural stem cells that induce pluripotent stem cells into spinal cord injury rats. The results showed that the cells grew well on the scaffold. In addition, the transplantation reduced the size of the lumen and improved the motor recovery ability of the rats. Tobias et al.[53] differentiated adipocytes into adipocytes and showed angiogenic activity by using an optimized alginate scaffold. The results showed that such scaffolds could significantly improve intravascular angiogenesis in eggs. $\mathrm{Xu}$ et al.[54] successfully promoted the adhesion, proliferation and angiogenesis of human umbilical vein endothelial cells on the scaffold by using heparinmodified endothelial acellular scaffold, which can be used to solve the problem of blood supply and better support the function of insulin-secreting cells. By injecting SDS solution, deionized water and polyethylene glycol octyl phenyl ether, $\mathrm{Du}$ et al.[55] obtained acellular renal scaffold that preserved a large number of natural microstructures and extracellular matrix, cultured renal progenitor cells and endothelial cells, and successfully completed the construction of glomerulus.

\section{Conclusion and Prospect}

This paper mainly introduces the research progress of three core elements of tissue engineering in the application of vital organs -- seed cells, growth factors and biomimetic scaffold materials. Although researchers have made a series of advances in the construction and repair of multiple organ tissues, there are still many challenges to realize the engineering-based construction of tissue organs for clinical organ injury transplantation. The source and differentiation efficiency of seed cells, the toxicity and side effects of growth factors, the safety of biomimetic scaffold materials, immune prototypes and clinical applications have not been properly solved. The following research should focus on the following issues: (1) how to further improve the function and stability of controllable differentiation of stem cells; (2) how to screen and apply growth factors safely and efficiently so as to target the damaged target cells; (3) how to solve the problems of difficult to control the degradation and absorption time of scaffold materials, uneven absorption and residue, find out the time, frequency and optimal cell concentration of scaffold injection, and reduce the possibility of stimulating host immune response. The solutions of these problems in the future will help to construct a complete and functional engineered tissue organs in vitro and lay a solid theoretical and technical foundation for clinical treatment of organ failure and injury. As more and more researchers have been devoting themselves to the research of tissue engineering and the further exploration of stem cell technology, biotechnology and chemical materials, more and more technical barriers will be broken. Tissue engineering-related techniques will also be used in the clinical treatment of severe organ diseases such as endstage liver disease, heart failure, myocardial infarction, chronic kidney disease and acute kidney injury.

\section{Reference}

1. Virani, S.S., et al., Heart Disease and Stroke Statistics-2020 Update: A Report From the American Heart Association. Circulation, 2020. 141(9): p. e139-e596.

2. Wang, M., et al., Improved osteogenesis and angiogenesis of magnesium-doped calcium phosphate cement via macrophage immunomodulation. Biomater Sci, 2016. 4(11): p. 1574-1583.

3. Gill, M.L., et al., Neuromodulation of lumbosacral spinal networks enables independent stepping after complete paraplegia. Nat Med, 2018. 24(11): p. 1677-1682.

4. Yu, J.R., et al., Current and Future Perspectives on Skin Tissue Engineering: Key Features of Biomedical Research, Translational Assessment, and Clinical Application. Adv Healthc Mater, 2019. 8(5): p. e1801471.

5. Noor, N., et al., 3D Printing of Personalized Thick and Perfusable Cardiac Patches and Hearts. Adv Sci (Weinh), 2019. 6(11): p. 1900344.

6. Ng, S.S., et al., Human iPS derived progenitors bioengineered into liver organoids using an inverted colloidal crystal poly (ethylene glycol) scaffold. Biomaterials, 2018. 182: p. 299-311.

7. Kaminski, M.M., et al., Direct reprogramming of fibroblasts into renal tubular epithelial cells by defined transcription factors. Nat Cell Biol, 2016. 18(12): p. 1269-1280.

8. Barcia Duran, J.G., et al., In vitro conversion of adult murine endothelial cells to hematopoietic stem cells. Nat Protoc, 2018. 13(12): p. 2758-2780.

9. Wang, Y., et al., Conversion of Human Gastric Epithelial Cells to Multipotent Endodermal Progenitors using Defined Small Molecules. Cell Stem Cell, 2016. 19(4): p. 449-461.

10. Bansal, V., et al., Chemical induced conversion of mouse fibroblasts and human adipose-derived stem cells into skeletal muscle-like cells. Biomaterials, 2019. 193: p. 30-46.

11. Cao, N., et al., Conversion of human fibroblasts into functional cardiomyocytes by small molecules. Science, 2016. 352(6290): p. 1216-20. 
12. Lim, K.T., et al., Direct Conversion of Mouse Fibroblasts into Cholangiocyte Progenitor Cells. Stem Cell Reports, 2018. 10(5): p. 1522-1536.

13. Yang, Y., et al., Rapid and Efficient Conversion of Human Fibroblasts into Functional Neurons by Small Molecules. Stem Cell Reports, 2019. 13(5): p. 862-876.

14. Zhu, Y., et al., Direct conversion of human myoblasts into brown-like adipocytes by engineered super-active PPARgamma. Obesity (Silver Spring), 2015. 23(5): p. 1014-21.

15. Takimoto, A., et al., Direct conversion of tenocytes into chondrocytes by Sox9. Exp Cell Res, 2012. 318(13): p. 1492-507.

16. Cassady, J.P., et al., Direct lineage conversion of adult mouse liver cells and B lymphocytes to neural stem cells. Stem Cell Reports, 2014. 3(6): p. 94856.

17. Si-Tayeb, K., et al., Highly efficient generation of human hepatocyte-like cells from induced pluripotent stem cells. Hepatology, 2010. 51(1): p. 297-305.

18. Umezawa, A., et al., Amnion-derived cells as a reliable resource for next-generation regenerative medicine. Placenta, 2019. 84: p. 50-56.

19. Heo, J.S., et al., Comparison of molecular profiles of human mesenchymal stem cells derived from bone marrow, umbilical cord blood, placenta and adipose tissue. Int J Mol Med, 2016. 37(1): p. 11525.

20. Pavathuparambil Abdul Manaph, N., et al., An overview on small molecule-induced differentiation of mesenchymal stem cells into beta cells for diabetic therapy. Stem Cell Res Ther, 2019. 10(1): p. 293.

21. Dabrowski, F.A., et al., Mesenchymal Stem Cells from Human Amniotic Membrane and Umbilical Cord Can Diminish Immunological Response in an in vitro Allograft Model. Gynecol Obstet Invest, 2017. 82(3): p. 267-275.

22. Mansoor, S.R., E. Zabihi, and M. GhasemiKasman, The potential use of mesenchymal stem cells for the treatment of multiple sclerosis. Life Sci, 2019. 235: p. 116830.

23. Bier, A., et al., Placenta-derived mesenchymal stromal cells and their exosomes exert therapeutic effects in Duchenne muscular dystrophy. Biomaterials, 2018. 174: p. 67-78.

24. Chulpanova, D.S., et al., Application of Mesenchymal Stem Cells for Therapeutic Agent Delivery in Anti-tumor Treatment. Front Pharmacol, 2018. 9: p. 259.

25. Munir, F., et al., Current status of diagnosis and Mesenchymal stem cells therapy for acute pancreatitis. Physiol Rep, 2019. 7(21): p. e14170.

26. Baranek, M., W.T. Markiewicz, and J. Barciszewski, Selected small molecules as inducers of pluripotency. Acta Biochim Pol, 2016. 63(4): p. 709-716.

27. Samavarchi-Tehrani, P., et al., Functional genomics reveals a BMP-driven mesenchymal-toepithelial transition in the initiation of somatic cell reprogramming. Cell Stem Cell, 2010. 7(1): p. 6477.

28. Li, R., et al., A mesenchymal-to-epithelial transition initiates and is required for the nuclear reprogramming of mouse fibroblasts. Cell Stem Cell, 2010. 7(1): p. 51-63.

29. Yang, J., et al., Stat3 activation is limiting for reprogramming to ground state pluripotency. Cell Stem Cell, 2010. 7(3): p. 319-28.

30. Huang, K., et al., Dynamically reorganized chromatin is the key for the reprogramming of somatic cells to pluripotent cells. Sci Rep, 2015. 5: p. 17691.

31. Li, D., et al., Optimized Approaches for Generation of Integration-free iPSCs from Human UrineDerived Cells with Small Molecules and Autologous Feeder. Stem Cell Reports, 2016. 6(5): p. 717-728.

32. Liu, K., et al., ATG3-dependent autophagy mediates mitochondrial homeostasis in pluripotency acquirement and maintenance. Autophagy, 2016. 12(11): p. 2000-2008.

33. Ma, T., et al., Atg5-independent autophagy regulates mitochondrial clearance and is essential for iPSC reprogramming. Nat Cell Biol, 2015. 17(11): p. 1379-87.

34. Qin, H., A. Zhao, and X. Fu, Small molecules for reprogramming and transdifferentiation. Cell Mol Life Sci, 2017. 74(19): p. 3553-3575.

35. Tang, Y., et al., Direct Conversion of Mouse Fibroblasts into Neural Stem Cells by Chemical Cocktail Requires Stepwise Activation of Growth Factors and Nup210. Cell Rep, 2018. 24(5): p. 1355-1362 e3.

36. Zhou, J., et al., Conversion of human fibroblasts into functional Leydig-like cells by small molecules and a single factor. Biochem Biophys Res Commun, 2019. 516(1): p. 1-7.

37. Liu, C., et al., Conversion of mouse fibroblasts into oligodendrocyte progenitor-like cells through a chemical approach. J Mol Cell Biol, 2019. 11(6): p. 489-495.

38. Kim, Y., et al., Small molecule-mediated reprogramming of human hepatocytes into bipotent progenitor cells. J Hepatol, 2019. 70(1): p. 97-107.

39. Ma, N.X., J.C. Yin, and G. Chen, Transcriptome Analysis of Small Molecule-Mediated Astrocyteto-Neuron Reprogramming. Front Cell Dev Biol, 2019. 7: p. 82.

40. Kang, H., et al., Small molecule-driven direct conversion of human pluripotent stem cells into functional osteoblasts. Sci Adv, 2016. 2(8): p. e1600691. 
41. Chen, Y., N. Kawazoe, and G. Chen, Preparation of dexamethasone-loaded biphasic calcium phosphate nanoparticles/collagen porous composite scaffolds for bone tissue engineering. Acta Biomater, 2018. 67: p. 341-353.

42. Asghari, F., et al., Biodegradable and biocompatible polymers for tissue engineering application: a review. Artif Cells Nanomed Biotechnol, 2017. 45(2): p. 185-192.

43. Rahman, M.S., et al., Fabrication of biocompatible porous scaffolds based on hydroxyapatite/collagen/chitosan composite for restoration of defected maxillofacial mandible bone. Prog Biomater, 2019. 8(3): p. 137-154.

44. Li, Q., et al., Hydroxyapatite/Collagen ThreeDimensional Printed Scaffolds and Their Osteogenic Effects on Human Bone MarrowDerived Mesenchymal Stem Cells. Tissue Eng Part A, 2019. 25(17-18): p. 1261-1271.

45. Yao, Q., et al., Electrospun collagen/poly(L-lactic acid-co-epsilon-caprolactone) scaffolds for conjunctival tissue engineering. Exp Ther Med, 2017. 14(5): p. 4141-4147.

46. Fu, W., et al., Electrospun gelatin/PCL and collagen/PLCL scaffolds for vascular tissue engineering. Int J Nanomedicine, 2014. 9: p. 233544.

47. Shu, Y., et al., RoY peptide-modified chitosanbased hydrogel to improve angiogenesis and cardiac repair under hypoxia. ACS Appl Mater Interfaces, 2015. 7(12): p. 6505-17.

48. $\mathrm{Wu}, \mathrm{Y}$., et al., Interwoven Aligned Conductive Nanofiber Yarn/Hydrogel Composite Scaffolds for Engineered 3D Cardiac Anisotropy. ACS Nano, 2017. 11(6): p. 5646-5659.

49. Lih, E., et al., Biomimetic Porous PLGA Scaffolds Incorporating Decellularized Extracellular Matrix for Kidney Tissue Regeneration. ACS Appl Mater Interfaces, 2016. 8(33): p. 21145-54.

50. Trivedi, S., et al., Hydroxyapatite-collagen augments osteogenic differentiation of dental pulp stem cells. Odontology, 2020. 108(2): p. 251-259.

51. Wu, Q., et al., The effect of heparinized decellularized scaffolds on angiogenic capability. J Biomed Mater Res A, 2016. 104(12): p. 3021-3030.

52. Zhou, X., et al., Polycaprolactone electrospun fiber scaffold loaded with iPSCs-NSCs and ASCs as a novel tissue engineering scaffold for the treatment of spinal cord injury. Int J Nanomedicine, 2018. 13: p. 6265-6277.

53. Hirsch, T., et al., Implant for autologous soft tissue reconstruction using an adipose-derived stem cellcolonized alginate scaffold. J Plast Reconstr Aesthet Surg, 2018. 71(1): p. 101-111.

54. Xu, L., et al., Constructing heparin-modified pancreatic decellularized scaffold to improve its reendothelialization. J Biomater Appl, 2018. 32(8): p. 1063-1070.
55. Du, C., et al., Functional Kidney Bioengineering with Pluripotent Stem-Cell-Derived Renal Progenitor Cells and Decellularized Kidney Scaffolds. Adv Healthc Mater, 2016. 5(16): p. 2080-91. 